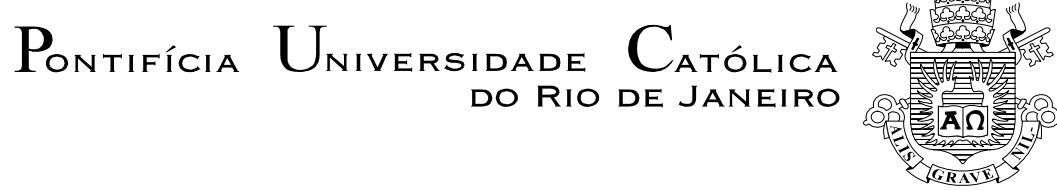

Anderson Luiz da Silva

LER(-SE) E ESCREVER(-SE) (N)O OUTRO

Tese de Doutorado

Tese apresentada ao Programa de Pósgraduação em Letras do Departamento de Letras da PUC-Rio como parte dos requisitos para obtenção do título de Doutor em Letras.

Orientadora: Pina Maria Arnoldi Coco 


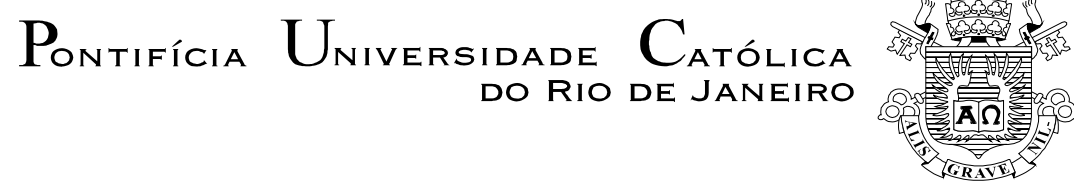

Anderson Luiz da Silva

\section{LER(-SE) E ESCREVER(-SE) (N)O OUTRO}

Tese apresentada como requisito parcial para obtenção do grau de Doutor pelo programa de Pós-Graduação em Letras do Departamento de Letras do Centro de Teologia e Ciências Humanas da PUC-Rio. Aprovada pela Comissão Examinadora abaixo assinada.

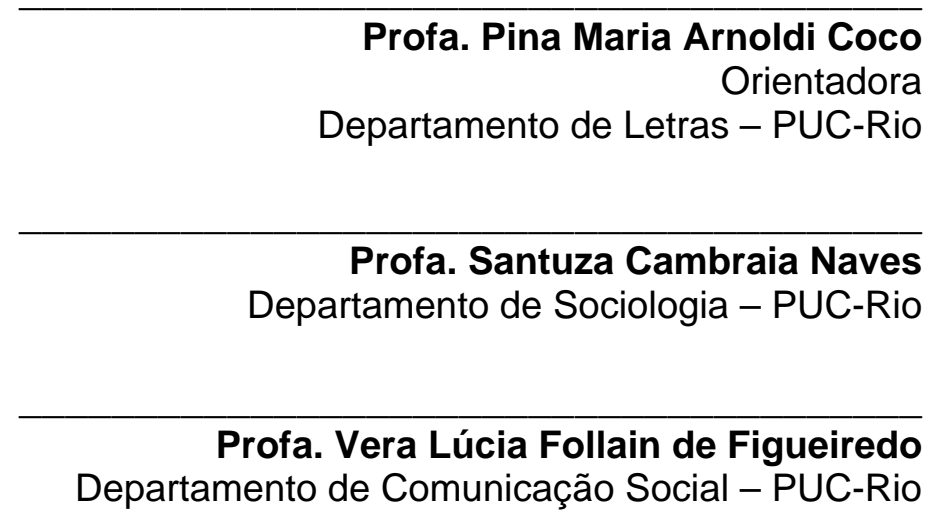

$\overline{\text { Profa. Daniela Gianna Claudia Beccaccia Versiani }}$ UNESA

Prof. Alexandre Graça Faria

UFJF

Prof. Paulo Fernando Carneiro de Andrade

Coordenador Setorial do Centro de Teologia

e Ciências Humanas - PUC-Rio

Rio de Janeiro, 29 de agosto de 2006 
Todos os direitos reservados. É proibida a reprodução total ou parcial do trabalho sem autorização da universidade, do autor e do orientador.

\section{Anderson Luiz da Silva}

Graduou-se em Letras na Universidade Federal de Juiz de Fora em 1994. Concluiu Especialização em Metodologia do Ensino Superior, no CES-Juiz de Fora(1995) e Mestrado em Literatura Brasileira, na PUCRio (1998). Atua, desde 1999, no magistério superior, especialmente nos cursos de Letras e Pedagogia.

Ficha Catalográfica

Silva, Anderson Luiz da

Ler(-se) e escrever(-se) (n)o outro / Anderson Luiz da Silva ; orientador: Pina Maria Arnoldi Coco. - 2006.

$183 \mathrm{f.} ; 30 \mathrm{~cm}$

Tese (Doutorado em Letras)-Pontifícia Universidade Católica do Rio de Janeiro, Rio de Janeiro, 2006.

Inclui bibliografia

1. Letras - Teses. 2. Literatura brasileira. 3. Século XX. 4. Prosa de ficção. 5. Alteridade. 6. Vozes etnográficas. I. Coco, Pina Maria Arnoldi. II. Pontifícia Universidade Católica do Rio de Janeiro. Departamento de Letras. III. Título. 


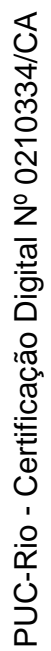

Ainda uma vez, à memória de meu pai. 


\section{Agradecimentos}

À Pina, por muito mais que orientar: confiar, acreditar e fazer-me acreditar que existe um possível, apesar de tudo.

Aos professores do Departamento de Letras da PUC-Rio, cujas aulas e conversas tanto colaboraram na construção de meu pensamento.

À Chiquinha, presença mais que profissional e competente, porque sempre solícita, carinhosa e amiga.

À Leda, minha mãe, e à Lu, minha irmã, por tanto e por tudo.

À Brenda, pela intensidade.

À tia Neném, pelas presenças e ausências nas horas certas.

À tia Zezé, Valéria e Édio, pelo apoio logístico e pelos tantos dias, noites e madrugadas de conversas, de que já tenho saudades.

Ao Lincoln, por ter se tornado mais que Amigo; Irmão.

À Valéria, pela amizade e pelas tantas interlocuções.

Ao André, paciente “ouvidor” e interlocutor de minhas vozes.

À Helena, pela fé e pela sensibilidade que transbordam no abraço de cada encontro.

À Maria Luíza, por tanta ajuda e amizade demonstradas nos últimos anos.

A todos os amigos que, cada um a seu modo, fazem-se presentes em meu caminho.

Às Professoras - no melhor sentido que o termo possa evocar - que me ensinaram a ser o que sou: Dona Neuza, Terezinha Scher, Enilce, Nil e, mais uma vez, Pina.

Aos que, por motivos tantos, o espaço não permite próximos, mas o tempo sim, pois que me habitam, e suas vozes se ouvem nas palavras que me escrevem. 


\section{Resumo}

SILVA, Anderson Luiz da; Coco, Pina Maria Arnoldi. Ler(-se) e escrever (-se) (n)o outro. Rio de Janeiro, 2006. 183p. Tese de doutorado Departamento de Letras. Pontifícia Universidade Católica do Rio de Janeiro.

O objetivo deste trabalho é discutir, na prosa de ficção brasileira da década de 1990, a presença de estratégias discursivas que revelam processos complexos de abordagem do confronto entre o eu e o outro. Trata-se da presença de instâncias narrativas impregnadas de discursos sobre a alteridade, o que se evidencia em contos e romances cujos enredos pautamse no confronto de subjetividades, etnias e culturas. Para discutir essa questão, procuramos identificar, nas narrativas analisadas, o que denominamos como a presença de vozes etnográficas, a partir de aproximações entre o campo da literatura e da antropologia, considerando que o procedimento de leitura e escrita do outro faz-se presente tanto no trabalho do etnógrafo quanto do ficcionista. Tal procedimento leva-nos, enfim, a considerar a polifonia como um traço fundamental do processo de escrita e leitura da alteridade em nossa ficção contemporânea.

\section{Palavras-chave:}

Literatura brasileira; prosa de ficção; década de 1990; alteridade; vozes etnográficas; polifonia. 


\section{Abstract}

SILVA, Anderson Luiz da; Coco, Pina Maria Arnoldi (Advisor). Read (himself) and write (himself) (in) the Other. Rio de Janeiro, 2006. 183p. Tese de doutorado - Departamento de Letras. Pontifícia Universidade Católica do Rio de Janeiro.

The objective of this study is to discuss the presence, in 1990's Brazilian prose fiction, of discursive strategies that reveal complex processes of approaching the confrontation between the $I$ and the Other. We deal with the presence of narrative instances impregnated with speeches on alterity, what is evidenced in stories and romances whose plots are based on the confrontation of subjectivities, ethnic groups and cultures. In order to discuss this subject, we tried to identify, in the narratives analyzed, what we denominate the presence of ethnographic voices, starting with approximations among the literary and the anthropologic fields, considering that procedures of the other's reading and writing are made present as much in the ethnographer's as in the fictionist's work. Such procedure lets us, finally, consider the polyphony as a fundamental feature of the alterity's writing and reading process in our contemporary fiction.

\section{Key words}

Brazilian literature; prose fiction; the 1990's; alterity; ethnographic voices; polyphony. 


\section{Sumário}

1. Introdução 10

2. Afinando vozes 18

2.1. Antecedentes 19

2.2. Deslocamentos 29

2.3. Deslocando vozes 36

2.4. Vozes etnográficas 44

3. Eu e outro, aqui e lá

3.1. Nem Hércules nem Quasímodo 55

3.2. O riso 63

3.3. O texto 76

3.4. O ensaio 90

3.5. A viagem 98

4. Eu e outro: ficções 105

4.1. A nostalgia 106

4.2. Os esquecimentos 117

4.3. O sabor 124

4.4. A biografia 129

4.5. O plágio 138

5. Eu e outro: performances 148

5.1. O diário 149

5.2. O etnógrafo 157

6. Conclusão 168

7. Referências Bibliográficas 175 
Homo sum, humani hihil a me alienum puto.

Publius Terentius Afer

Eu sou trezentos, sou trezentos-e-cincoenta, Mas um dia afinal eu toparei comigo ...

Mário de Andrade

Aprendi que ninguém pode descobrir o labirinto em que o outro vive, sem se perder no próprio.

Jorge Andrade 